\title{
Research
}

Jo B Middlemass, Momina F Yazdani, Joe Kai, Penelope J Standen and Nadeem Qureshi

\section{Introducing genetic testing for cardiovascular disease in primary care:}

\author{
a qualitative study
}

\begin{abstract}
\section{Background}

While primary care systematically offers conventional cardiovascular risk assessment, genetic tests for coronary heart disease (CHD) are increasingly commercially available to patients. It is unclear how individuals may respond to these new sources of risk information.
\end{abstract}

\section{Aim}

To explore how patients who have had a recent conventional cardiovascular risk assessment, perceive additional information from genetic testing for $\mathrm{CHD}$

\section{Design and setting}

Qualitative interview study in 12 practices in Nottinghamshire from both urban and rural settings.

\section{Method}

Interviews were conducted with 29 adults, who consented to genetic testing after having had a conventional cardiovascular risk assessment.

\section{Results}

Individuals' principal motivation for genetic testing was their family history of $\mathrm{CHD}$ and a desire to convey the results to their children. After testing, however, there was limited recall of genetic test results and scepticism about the value of informing their children. Participants dealt with conflicting findings from the genetic test, family history, and conventional assessment by either focusing on genetic risk or environmental lifestyle factors. In some participants, genetic test results appeared to reinforce healthy behaviour but others were falsely reassured, despite having an 'aboveaverage' conventional cardiovascular risk score.

\section{Conclusion}

Although genetic testing was acceptable, participants were unclear how to interpret genetic risk results. To facilitate healthy behaviour, health professionals should explore patients' understanding of genetic test results in light of their family history and conventional risk assessment.

\section{Keywords}

coronary heart disease; family practice; genetic testing; primary health care.

\section{INTRODUCTION}

Cardiovascular disease (CVD) is the top cause of death globally; ${ }^{1}$ it causes a third of deaths worldwide ${ }^{2}$ and is projected to remain the single leading cause of death. ${ }^{3}$ To help identify individuals at increased risk of it, many clinicians use riskassessment tools, such as those using the Joint British Societies' (JBS2) and QRISK2 tools. ${ }^{4,5}$ The former is based on the Framingham algorithm to give a probability of developing a cardiovascular event within the next 10 years, and gives GPs a basis and rationale on which to prescribe medication to reduce overall CVD risk. ${ }^{6-8}$ The JBS2 tool and a few other cardiovascular risk assessment tools take account of a family history of premature $\mathrm{CHD}$ as a proxy for genetic predisposition. $4,5,7,9$

Recent genome studies have identified genetic variants, such as the $9 p 21$ gene, as strong independent risk factors for CHD. This information may have clinical utility in reclassifying $\mathrm{CHD}$ risk. ${ }^{10-12}$ At the same time, predictive genetic tests are increasingly being offered commercially, directly to consumers over the counter. ${ }^{13}$ The current limitations of this type of genetic testing are not always considered, with both participants and clinicians not fully understanding the test results. 14,15 This may lead either to false reassurance or unnecessary increased stress or anxiety. ${ }^{14}$

JB Middlemass, research fellow, MA, MPhil, PhD research assistant, School of Health and Social Care, University of Lincoln, Lincoln. MF Yazdani, BMedSci, medical student; J Kai, MD, FRCGP clinical professor of primary care; $\mathbf{N}$ Qureshi, DM, MRCGP, clinical professor of primary care, Division of Primary Care; PJ Standen, BSc, PhD professor in health psychology and learning disabilities, Division of Ageing and Rehabilitation, University of Nottingham, Nottingham.

\section{Address for correspondence}

Nadeem Qureshi, clinical professor of primary care, Division of Primary Care, University of
However, using genetic testing in primary care could be useful as a tool to encourage risk-reducing behaviour in those at risk of developing CVD. ${ }^{16-18}$ Arguably, this may be more effective than conventional cardiovascular risk assessment alone, as genetic risk may seem more personal and salient than family history. ${ }^{19}$

In this study, adults who had had a recent cardiovascular risk assessment in general practice were offered a genetic test for $\mathrm{CHD}$ to explore the acceptability and feasibility of the procedure and assessment. This article reports a qualitative exploration with participants on how they made sense of the additional information from genetic testing and their perceptions of whether this influenced their behaviour.

\section{METHOD}

\section{Design}

This qualitative interview study formed part of a wider mixed-methods study to evaluate the feasibility and clinical utility of genetic testing as part of conventional cardiovascular risk assessment.

\section{Participants}

The consenting participants came from 12 practices in Nottinghamshire from both urban and rural settings and had had a conventional cardiovascular risk assessment within the last 18 months. ${ }^{5}$
Nottingham, 13th Floor, Tower Building, University Park, Nottingham NG7 2RD.

E-mail: nadeem.qureshidnottingham.ac.uk

Submitted: 11 November 2013; Editor's response: 2 December 2013; final acceptance: 9 February 2014

\section{@British Journal of General Practice}

This is the full-length article (published online 28 Apr 2014) of an abridged version published in print. Cite this article as: Br J Gen Pract 2014; DOI: 10.3399/bjgp14X679714. 


\section{How this fits in}

Cardiovascular risk assessment using conventional risk factors is routine in primary care but 'direct-to-consumer' genetic testing for coronary heart disease (CHD) is now easily available, particularly online. This study indicates that uptake of genetic testing for $\mathrm{CHD}$ may be particularly attractive to patients with a family history of the condition. When encountering individuals who have had genetic testing, GPs need to explore the individual's understanding of the results in light of their family history and the previous conventional risk assessment to ensure adherence with risk-reducing behaviour.

They were individuals who had, previously, received their conventional cardiovascular risk assessment and had chosen to have a genetic test, as well as agreeing to a followup interview. The interviews were carried out 4 months after patients received their risk status. On recruitment, the study pack included a family-history questionnaire enquiring about CHD in first- and seconddegree relatives.

The CHD genetic test lwhich is commercially available) involved collecting a saliva sample from the participant and sending it for analysis to University College London's cardiovascular genetic laboratory. It comprised a cardiovascular panel of nine risk alleles to produce a combined risk profile score.

Participants were sent a letter outlining the results of their conventional risk assessment, genetic test, and their overall cardiovascular risk. The letter presented both the conventional assessment and genetic test results in terms of average and above-average risk. In line with other risk-assessment tools, these were classified as follows:

- average risk; at or near population risk; and

- above-average; greater than population risk.

In the conventional cardiovascular risk assessment, average was $<10 \%$ risk of developing CVD in the next 10 years and above average was $\geq 10 \%$ risk. ${ }^{4,5}$

To avoid the study interfering with participants' adherence to current CVD risk-assessment recommendations, ${ }^{5}$ if the conventional cardiovascular risk assessment suggested above-average risk and the genetic test indicated that risk was average, participants were advised that they were still at above-average risk of developing CVD.

\section{Interview schedule}

Participants were asked about their:

- conventional risk assessment, which had been undertaken at the practice;

- experience of the genetic test; and

- interpretations and perceptions of the results of both assessments.

Their views on whether results had or had not influenced any change in their behaviours over the 4 months of the study were also explored.

\section{Analytical approach}

All interviews were transcribed verbatim. Interview data were managed using Atlas. ti (version 5). Thematic analysis was used, ${ }^{20}$ and codes and themes developed iteratively as data were analysed. ${ }^{21}$ Codes were both deductive (derived from the interview schedule) and inductive (data driven).

\section{RESULTS}

A total of 320 individuals who had had a conventional CVD risk assessment were offered genetic testing. Of those, 119 agreed to have the test and 30 consented to be interviewed; one potential participant was excluded from the interview because of psychosocial reasons.

Table 1 outlines the participants characteristics for both the main feasibility study and this qualitative study. Like the main study, most participants were males in their mid-50s to mid-60s, while in the qualitative study $41 \%$ had qualifications equivalent to, or greater than, A-levels. Of the 29 participants, seven had genetic test results that showed above-average risk, with four of these reporting a family history of CHD. The genetic test results of the remaining 22 participants showed they were at average risk; 13 of these had recalled a family history of $\mathrm{CHD}$.

The age range of the 119 participants in the main feasibility study and the 29 interviewees was similar (median 59 years; interquartile range [IQR] 53-62 years). Proportionally, more males $(72.4 \%$ versus $58.0 \%$ ) and more individuals who were educated to undergraduate degree level $134.5 \%$ versus $18.5 \%$ ) participated in the interviews.

In terms of being categorised differently in the conventional assessment and the genetic test, when compared with all the study participants, more from the qualitative study 


\section{Table 1. Participants' characteristics}

\begin{tabular}{|c|c|c|}
\hline & $\begin{array}{l}\text { Feasibility study, } \\
\qquad n=119(\%)^{\mathrm{a}}\end{array}$ & $\begin{array}{c}\text { Qualitative study, } \\
\text { subsample, } n=29(\%)^{a}\end{array}$ \\
\hline Excluded participants & $1(0.84)$ & 0 \\
\hline Age in years, median (IQR) & 59 (53-62) & $59(53.5-62)$ \\
\hline Male sex & 69 (58.0) & $21(72.4)$ \\
\hline \multicolumn{3}{|l|}{ Ethnic group } \\
\hline $\begin{array}{l}\text { White } \\
\text { Asian } \\
\text { Mediterranean } \\
\text { Missing }\end{array}$ & $\begin{array}{c}96(80.7) \\
1(0.8) \\
1(0.8) \\
21(17.6)\end{array}$ & $\begin{array}{c}28(96.6) \\
0(0.0) \\
1(3.4) \\
0(0.0)\end{array}$ \\
\hline \multicolumn{3}{|l|}{ Educational/professional qualification } \\
\hline $\begin{array}{l}\text { GCSE/O-level/CSE } \\
\text { Vocational qualification } \\
\text { A-level or equivalent } \\
\text { First degree } \\
\text { Other } \\
\text { No formal qualifications } \\
\text { Still studying } \\
\text { Missing }\end{array}$ & $\begin{array}{l}14(11.8) \\
6(5.0) \\
14(11.8) \\
22(18.5) \\
21(17.6) \\
17(14.3) \\
1(0.8) \\
24(20.2)\end{array}$ & $\begin{array}{c}2(6.9) \\
3(10.3) \\
2(6.9) \\
10(34.5) \\
5(17.2) \\
6(20.7) \\
0(0.0) \\
1(3.4)\end{array}$ \\
\hline \multicolumn{3}{|l|}{ CVD risk score } \\
\hline $\begin{array}{l}\text { Average risk ( }<10 \%, 10 \text {-year CVD risk) } \\
\text { Moderate risk (10-19\% 10-year CVD risk) } \\
\text { High risk (20\%+, 10-year CVD risk) }\end{array}$ & $\begin{array}{l}40(33.6) \\
53(44.5) \\
26(21.8)\end{array}$ & $\begin{array}{c}6(20.7) \\
18(62.1) \\
5(17.2)\end{array}$ \\
\hline
\end{tabular}

\section{CVD risk assessment/genetic test}

CVD risk assessment above average

with average genetic test

CVD risk assessment average but

genetic test above-average

CVD risk assessment above average,

with above-average genetic test

CVD risk assessment average with average genetic test

Unless specified. CSE = Certificate of Secondary Education. $C H D=$ coronary heart disease. $C V D=$ cardiovascular disease. GCSE = General Certificate of Secondary Education. IQR = interquartile range. testing was a family history that they wished to clarify further. For some, this related to a specific relationship - for example a parent or sibling with CHD - whereas, for others, it concerned relatives scattered across several generations.

Anxiety about premature death due to family history was expressed by only a minority of participants, with some saying this subsided when they had passed the age of their relative's death:

Well my father died aged 49 with coronary heart, you know, coronary thrombosis, so I just assumed there might be something in the family, something genetic and it was very difficult when I reached that age ... I was thinking, you know, "is this my time up?" But obviously it wasn't thankfully. (Participant 12, male, 62 years, aboveaverage CVD assessment result, average genetic test result, CHD family history, educated to degree level)

... and my grandfather on his side was, er, suffered with heart problems ... which really made me think if I don't look after what is going off, I need to be sort of sure of what is happening and, if it is going to be genetic, then it's going to come my way. '(Participant 24, male, 63 years, aboveaverage CVD assessment result, average genetic test result, CHD family history, educated to degree level)

Benefit to children. Responders also reported being tested to be able to discuss the outcome with their children. They felt the results would be useful for their offspring, who could make informed decisions if it was felt they should consider ways to reduce their own risk of developing $\mathrm{CHD}$ in the future:

'The reason I was interested ... was because my dad died in 1989 ... I believe it was the cardiovascular but I don't know. He was 62. It's young isn't it? So, obviously, I have got children of my own and so I need to make sure that it's not something that I am likely to pass on or have already passed on ... So all I am interested in, in reality, is protecting my kids and myself. And I think through this genetic thing we should be able to do it hopefully.' (Participant 4, male, 57 years, above-average CVD assessment result, average genetic test result, CHD family history, educated to vocational levell

However, at interview, very few participants had made their children aware of the genetic test. Although children were 
often adults, participants still felt they were not old enough to be concerned about CHD, or genetic testing:

'Erm they [adult children] haven't shown particular concern [...] no / think they all feel they are fairly healthy.' (Participant 12 male, 62 years, above-average CVD risk assessment result, average genetic test result, CHD family history, educated to degree level)

'No, no, no, no, you know they are 34 and 32. They are not, sort of... no never mentioned anything.' (Participant 1, male, 65 years, above-average CVD assessment result, average genetic test result, CHD family history, educated to degree levell

\section{Recall and initial reaction}

A number of participants could not initially recall receiving the genetic test and/or the cardiovascular risk assessment results, or what these had indicated:

I may be wrong but I can't remember ever having anything back.' (Participant 25, male, 54 years, above-average CVD assessment result, average genetic test result, CHD family history, no formal qualifications)

Some had to be reminded of the test results during interview. Upon receiving their results, some participants were confused by the 'average' and 'above-average' categories used to describe their risk. They had assumed there were further categories of increasing risk, beyond the dichotomous categorisation of 'average' and 'above average', to which they might have attached more importance:

'If it had come out as 'extremely high' I would have looked at it and thought "Blimey, what do I need to do, what does that mean? Should I be ringing someone?" Erm... but, yes, I suppose it is half reassuring. (Participant 17, male, 36 years, average CVD risk assessment result, above-average genetic test result, CHD family history, educated to degree level)

\section{Reconciling conflicting information}

Some participants rationalised their average genetic result, whilst having a significant family history, by reasoning that the genetic test was more important than their reported family history of $\mathrm{CHD}$

'Yes, I think I was [surprised] because I was sure that there was something in the family make-up, genetic make-up, but it's nice to know that it's not the case.' (Participant 12 , male, 62 years, above-average CVD risk assessment result, average genetic test result, CHD family history, educated to degree levell

Those whose risk was classified as 'average' in the conventional risk assessment but above average in the genetic test also felt that the genetic test was more important:

'I think it's different because you don't know which genes you have inherited ... so just because you have got family history ... It gives you a probability of having inherited that risk ... It doesn't definitely mean you have, whereas if you have a genetic test and genetically you have that high risk ... (Participant 16, female, 42 years, average CVD risk assessment result, above-average genetic test result, CHD family history, 'other' education)

It was noted that family history, as well as identifying genetic risk factors, incorporates ancestors' environmental risk factors, which the participant could modify or avoid:

.... it seems as if I am not following in my ancestors' footpath ... because if I am above-average [genetic risk] now, [but average CVD risk], whatever I have done up to now has been good, so let's improve on it, you know. Let's see if we can't make that better.' (Participant 19, female, 65 years, average CVD risk assessment result, above-average genetic test result, recalled CHD family history, 'other' education)

Lifestyle was seen by some responders as impacting on risk, independent of genetic inheritance, suggesting an appreciation of gene-environment interaction:

'Well it's obviously the genetics ... hasn't gone down through the family and I am just like an everyday person but, obviously, with the lifestyle / have led, or lead, [it] puts me at greater risk than the person who didn't live my lifestyle.' (Participant 3, male, 62 years, above-average CVD risk assessment result, average genetic test result, CHD family history, educated to A-level standard)

Others were sceptical of the value of genetic testing, questioning the relevance of the test results to themselves and their offspring:

'I don't know whether these tests could prove positively that erm if I had got a 
problem would my son have a problem it can't be proved can it ... No I don't feel that anything is written in stone as far as genes are concerned. You know it's just one of those things that can happen and I live with that.' (Participant 4, male, 57 years, above-average CVD risk assessment result, average genetic test result, CHD family history, educated to vocational levell

\section{Responses to genetic test result}

Behavioural change. An above-average result from the genetic test was, perhaps surprisingly, positively received by many participants as it confirmed their preconceived beliefs about familial heart disease risk and could push them to try to reduce that risk:

It hasn't worried me particularly, I think I've just carried on. But it's just given you another awareness really, I suppose, and, hopefully, try a little bit harder to do the right thing.' (Participant 5, female, 61 years, average CVD risk assessment result, aboveaverage genetic test result, CHD family history, educated to A-level standard)

Participants expressed the benefit of using the result to motivate behaviour change, particularly when there was concordance between the results of the genetic test and the conventional risk assessment:

I think the two together, erm, the two bits of information just help create a greater incentive for me to change things. (Participant 21, male, 53 years, aboveaverage CVD risk assessment result, above-average genetic test result, no CHD family history, 'other' education)

Most participants reported already undertaking lifestyle risk-reducing behaviour, however, downplaying the fact that the genetic test result might influence their behaviour:

Well I don't know what more changes I could make, except possibly exercising more, going to a gym or something like that. Er, I don't know. I would if I thought I was living an unhealthy lifestyle. But I don't think I am living an unhealthy lifestyle. (Participant 29, female, 64 years, average CVD risk assessment result, above-average genetic test result, CHD family history, educated to degree level)

False reassurance. In some cases, despite an above-average conventional risk assessment score, an average genetic test result appeared to falsely reassure participants that they did not have to modify their lifestyle, for example, so they remained disinclined to stop smoking. Similarly, an average genetic test result did not lead to the modification of a fatalistic attitude towards developing CHD. The individual attributed these concerns to a poor family history rather than increased risk on conventional assessment:

'If it's going to run in the family, you have got to accept it haven't you? If it's your turn to, if your number comes up, you can't do nothing about it.' (Participant 25, male, 54 years, above-average CVD risk assessment result, average genetic test result, CHD family history, no formal qualifications')

It was uncommon for participants to describe experiencing anxiety after receiving their genetic test result but, where this was expressed, it was seen as a motivator to reduce risk:

'Well, it scares me ... Erm, well, obviously, I want to do everything I possibly can to avoid, erm, to avoid getting any heart disease. (Participant 16, female, 42 years, average CVD risk assessment result, above-average genetic test result, CHD family history, 'other' education)

\section{Future role of genetic testing}

Testing using a saliva sample was considered to be acceptable to participants; none expressed concerns about the procedure. Some suggested this should be offered to the general public:

Er, no I think it ought to be available to everybody if they want to do it. Some people might think "I would rather not know".' (Participant 3, male, 62 years, above-average CVD risk assessment result, average genetic test result, CHD family history, educated to A levell

An older participant made the point that a genetic test was more appropriate for a younger age group, in whom preventative activities were more likely to lead to potential health benefits:

I think 25 ... At least it would point to them and, er, give them plenty of time to adjust to the lifestyle - if they want to, of course ...' (Participant 3, male, 62 years, above-average CVD risk assessment result, average genetic test result, CHD family history, educated to A-level standard) 
Others expressed concerns about relying on genetic test results and said that more research was required to identify further predictive genes for $\mathrm{CHD}$ :

'There will be a load of other genes that you don't know about yet... I haven't interpreted to mean that I haven't got any genes that are involved that might increase my cardiovascular risk you know. I don't take it to mean that it's all environmental ... I think there are probably other genes that will affect one's cholesterol, metabolism, and one's blood pressure, or whatever, that you perhaps haven't mapped yet.' (Participant 23, female, 53 years, above-average CVD risk assessment result, average genetic test result, CHD family history, 'other' education]

A participant who had experience with screening for another medical condition highlighted concerns that genetic testing may lead to insurance liabilities and break confidentiality:

'The only reason I took part in this genetic test was because it was guaranteed to be confidential and not passed on to any other party. '(Participant 20, male, 57 years, average CVD risk assessment result, above-average genetic test result, reports CHD family history, educated to GCSE/Olevel/CSE levell)

\section{DISCUSSION}

\section{Summary}

The principal driver for undertaking genetic testing was a perceived familial risk of developing $\mathrm{CHD}$, with participants often motivated by a desire to convey the results to their children. After testing, however some participants demonstrated limited recall of their results and scepticism about the value of passing the information to their offspring.

Participants had to deal with the fact that sometimes results from the genetic test, family history, and conventional cardiovascular risk assessment conflicted with each other. For some participants, the ways of dealing with this included either focusing on the genetic risk or the environmental lifestyle risk factors; others were sceptical about the value of the genetic test results.

In some participants the genetic test results appeared to reinforce healthy behaviour; others were falsely reassured by having an average score despite their conventional cardiovascular risk score being above average.
Participants found the testing procedure acceptable, and suggestions were made that genetic testing could lead to potential benefits for younger individuals by encouraging them to adopt risk-reducing behaviour.

\section{Strengths and limitations}

To the study's knowledge, this is the first study to explore the impact of genetic testing in individuals who have been offered a CVD risk assessment in primary care. Previous studies have only explored the reactions of participants to hypothetical genetic testing scenarios. ${ }^{22}$

This qualitative study explored the views of a self-selected sample of people who consented to genetic testing and followup interview. Although the participants were recruited from all of those who were undergoing cardiovascular risk assessment in a range of British general practices, there was limited participation by younger individuals, those from minority ethnic groups, and those from areas of deprivation.

Compared with all participants in the main feasibility study, proportionally more interviewees had an above-average conventional assessment result and an average genetic test result. However, the views of participants with a wide range of conventional and genetic cardiovascular risk profiles were captured.

Participants struggled to recall their genetic test results and had to be reminded of these during the interview; however, they were still able to give reasonable insight into their reaction to the results. This suggests that patients should be provided with more detailed information in the results letter.

The study had limited opportunity to examine any reported impact of genetic testing on behavioural change as many participants said they had already adopted healthy lifestyles prior to this study being undertaken.

\section{Comparison with existing literature}

To the study's knowledge this is the first study to evaluate patients' perspectives of genetic testing in the context of cardiovascular risk assessment in primary care. The majority of previous studies have explored the role of genetic testing in assessing the risk of specific monogenic diseases such as Huntington's disease, familial hypercholesterolaemia, and, more generally, in genetic risk assessments for cancer. ${ }^{23-27}$ Participants' opinions have also been ascertained in hypothetical scenarios. ${ }^{28,29}$

In this study, a perceived familial risk 
of $\mathrm{CHD}$ was a major driver for requesting genetic testing. This parallels the findings in studies of heart disease and breast cancer in secondary care..$^{30-31}$ Similar to this study, Lerman et al noted that most participants were motivated to know whether this predisposition would affect others in the family. ${ }^{31}$ However, the study also found that, after testing, some participants expressed scepticism about the value of passing on this information to their children. Further, participants with average genetic risk results found it difficult to reconcile these results with knowing that they had a family history of CHD. This is similar to the reported impact of a negative genetic test result for familial hypercholesterolaemia, which led to participants feeling 'in limbo' about their raised blood cholesterol. ${ }^{23}$ In this study, fatalism was reported in one individual only. This limited discussion is similar to studies communicating genetic test results to participants with a family history of familial hypercholesterolaemia. 23,24

Previous studies exploring the value of genetic testing in modifying risk-reducing behaviour have been disappointing. For those who underwent genetic testing for diabetes, myocardial infarction, and cancer, there has been little or no effect on longterm abstinence of smoking or short-term changes in diet and exercise. $22,28,32$

There are concerns that negative genetic test results for CVD may lead to false reassurance, while positive results may induce unnecessary anxiety in others. ${ }^{33}$ In this study, the false reassurance was identified but not related to above-average results.

\section{Implications for practice}

GPs are currently struggling to interpret conventionalcardiovascularriskassessment scores and communicate the results to patients. ${ }^{34,35}$ Even if there was adequate analytical and clinical validity of these CHD genetic tests, ${ }^{36}$ until the fundamental issues of clinicians' confidence in interpreting and communicating cardiovascular risk are resolved, incorporating genetic test results may be premature. ${ }^{35}$ However, GPs are exposed to genetic testing by patients presenting to discuss the results of commercially available (over-the-counter) CHD genetic tests. ${ }^{14}$ GPs will usually be the first point of contact and will need to explore the patient's understanding of potentially conflicting results from genetic testing and conventional cardiovascular risk assessments. However, many clinicians indicate a lack of awareness on the subject and feel unprepared to manage such patients. ${ }^{37}$

Further, in the future, genetic testing may be incorporated into primary care as a way of personalising the conventional cardiovascular risk assessment. In this instance, consideration will need to be given to the confidentiality of this information. . $^{1533,38}$ Although genetic testing in isolation may have limited utility, a high genetic risk score, in combination with a positive family history and an above-average conventional CVD risk assessment, may indicate a subgroup of the general population at particularly high risk of developing CVD and should be targeted for intensive lifestyle advice and lipid-lowering therapy.

Prior to implementing further research on the clinical utility of CHD genetic testing, further exploration of the best way to present the results to participants should be undertaken, as participants in this study did not understand the 'average' and 'above-average' classifications.

Any follow-up study might usefully involve a younger population and, as such, not be anchored to only those undergoing cardiovascular risk assessment (who are, in general, aged >40 years). To improve generalisability, future studies should actively recruit participants from various backgrounds including minority ethnic communities and groups who are underserved. It would also be beneficial to follow the participants over a longer period of time to see whether intentions to change behaviours did lead to actual behavioural changes.

\section{CONCLUSION}

These results add to the existing knowledge about patients' perceptions and understanding of their CHD risk, as identified by genetic testing. Participants had no concerns about testing procedures but, as they may make different judgements about their perceived risk of developing CVD in the light of their genetic risk result, health professionals need to explore patients' understanding of the conventional cardiovascular risk assessment, their perceived family history, commercially available genetic tests, and the impact of any discrepancy between the test and risk assessment on risk-reducing behaviour.

\section{Discuss this article}

Contribute and read comments about this article: www.bjgp.org/letters 


\section{REFERENCES}

1. World Health Organization. Global status report on noncommunicable diseases 2010. http://www.who.int/nmh/publications/ncd_report_full_en.pdf laccessed 14 Apr 2014).

2. WHO, World Heart Federation, and World Stroke Organization. Global atlas on cardiovascular disease prevention and control. Policies, strategies and interventions. Geneva: World Health Organization, 2011. http://www.who.int/ cardiovascular_diseases/publications/atlas_cvd/en/ (accessed 14 Apr 2014).

3. Mathers CD, Loncar D. Projections of global mortality and burden of disease from 2002 to 2030. PLoS Med 2006; 3(11): e442.

4. Hippisley-Cox J, Coupland C, Robson J, Brindle P. Derivation, validation, and evaluation of a new QRISK model to estimate lifetime risk of cardiovascular disease: cohort study using QResearch database. BMJ 2010; 341: c6624.

5. Wood D, Wray R, Poulter N, Williams B, et al. JBS 2: Joint British Societies guidelines on prevention of cardiovascular disease in clinical practice. Heart 2005; 91 (suppl. 5): vi-v52.

6. Mendis S. The contribution of the Framingham Heart Study to the prevention of cardiovascular disease: a global perspective. Prog Cardiovasc Dis 2010; 53(1): $10-14$.

7. Ridker PM, Buring JE, Rifai N, Cook NR. Development and validation of improved algorithms for the assessment of global cardiovascular risk in women: the Reynolds Risk Score. JAMA 2007; 297(6): 611-619.

8. Anderson KM, Odell PM, Wilson PW, Kannel WB. Cardiovascular disease risk profiles. Am Heart J 1991; 121(1 Pt 2): 293-298

9. Woodward M, Brindle P, Tunstall-Pedoe H, SIGN group on risk estimation. Adding social deprivation and family history to cardiovascular risk assessment: the ASSIGN score from the Scottish Heart Health Extended Cohort (SHHEC). Heart 2007; 93: 172-176

10. Johansen CT, Lanktree MB, Hegele RA. Translating genomic analyses into improved management of coronary artery disease. Future Cardiol 2010; 6(4): 507-521.

11. Talmud PJ, Cooper JA, Palmen J, et al. Chromosome 9p21.3 coronary heart disease locus genotype and prospective risk of $\mathrm{CHD}$ in healthy middle-aged men. Clin Chem 2008; 54(3): 467-474.

12. Swerdlow DI, Holmes MV, Harrison S, Humphries SE. The genetics of coronary heart disease. Br Med Bull 2012; 102: 59-77.

13. Gollust SE, Hull SC, Wilfond BS. Limitations of direct-to-consumer advertising for clinical genetic testing. JAMA 2002; 288(14): 1762-1767.

14. Rafi I, Qureshi N, Lucassen A, et al. 'Over-the-counter' genetic testing: what does it really mean for primary care? Br J Gen Pract 2009; DOI: 10.3399/bjgp09X395021.

15. Skirton H, Goldsmith L, Jackson L, O'Connor A. Direct to consumer genetic testing: a systematic review of position statements, policies and recommendations. Clin Genet 2012; 82(3): 210-218

16. McBride CM, Koehly LM, Sanderson SC, Kaphingst KA. The behavioral response to personalized genetic information: will genetic risk profiles motivate individuals and families to choose more healthful behaviors? Ann Rev Public Health 2010; 31 89-103.

17. Collins FS, Green ED, Guttmacher AE, Guyer MS, Institute UNHGR. A vision for the future of genomics research. Nature 2003; 422(6934): 835-847.

18. O'Neill SC, Lipkus IM, Sanderson SC, et al. Motivations for genetic testing for lung cancer risk among young smokers. Tobacco control 2013; 22(6): 406-411.

19. McBride CM, Bepler G, Lipkus IM, et al. Incorporating genetic susceptibility feedback into a smoking cessation program for African-American smokers with low income. Cancer Epidemiol Biomarkers Prev 2002; 11(6): 521-528.

20. Braun V, Clarke V. Using thematic analysis in psychology. Qual Res Psychol 2006 : 3(2): 77-101.

21. Barbour RS, Kitzinger J. Developing focus group research: politics, theory and practice. London: Sage ,1999.

22. Marteau TM, French DP, Griffin SJ, et al. Effects of communicating DNA-based disease risk estimates on risk-reducing behaviours. Cochrane Database Syst Rev 2010; (10): CD007275

23. Hilgart J, Mercer J, Thirlaway K. Individuals' experiences of, and responses to, a negative genetic test result for familial hypercholesterolaemia. J Health Psychol 2013; 18(3): 339-349.

24. Marteau T, Senior V, Humphries SE, et al. Psychological impact of genetic testing for familial hypercholesterolemia within a previously aware population: a randomized controlled trial. Am J Med Genet A 2004; 128A(3): 285-293.

25. Dorval M, Gauthier G, Maunsell E, et al. No evidence of false reassurance among women with an inconclusive BRCA1/2 genetic test result. Cancer Epidemiol Biomarkers Prev 2005; 14(12): 2862-2867.

26. Johnson K, Trimbath J, Petersen G, et al. Impact of genetic counseling and testing on colorectal cancer screening behavior. GenetTest 2002; 6(4): 303-306.

27. McBride CM, Halabi S, Bepler G, et al. Maximizing the motivational impact of feedback of lung cancer susceptibility on smokers' desire to quit. J Health Commun 2000; 5(3): 229-241.

28. Sanderson SC, Michie S. Genetic testing for heart disease susceptibility: potential impact on motivation to quit smoking. Clin Genet 2007; 71(6): 501-510.

29. Cameron LD, Sherman KA, Marteau TM, Brown PM. Impact of genetic risk information and type of disease on perceived risk, anticipated affect, and expected consequences of genetic tests. Health Psychol 2009; 28(3): 307-316.

30. Sanderson SC, Wardle J, Jarvis MJ, Humphries SE. Public interest in genetic testing for susceptibility to heart disease and cancer: a population-based survey in the UK. Prev Med 2004; 39(3): 458-464.

31. Lerman C, Croyle RT, Tercyak KP, Hamann H. Genetic testing: psychological aspects and implications. J Consult Clin Psychol 2002; 70(3): 784-797.

32. Bloss CS, Schork NJ, Topol EJ. Effect of direct-to-consumer genomewide profiling to assess disease risk. N Engl J Med 2011; 364(6): 524-534.

33. Humphries SE, Ridker PM, Talmud PJ. Genetic testing for cardiovascular disease susceptibility: a useful clinical management tool or possible misinformation? Arterioscler Thromb Vasc Biol 2004; 24(4): 628-636.

34. Kirby M, Machen I. Impact on clinical practice of the Joint British Societies cardiovascular risk assessment tools. Int J Clin Pract 2009; 63(12): 1683-1692

35. Liew SM, Blacklock C, Hislop J, et al. Cardiovascular risk scores: qualitative study of how primary care practitioners understand and use them. Br J Gen Pract 2013; DOI: 10.3399/bjgp13X668195

36. Teutsch SM, Bradley LA, Palomaki GE, et al. The Evaluation of Genomic Applications in Practice and Prevention (EGAPP) Initiative: methods of the EGAPP Working Group. Genet Med 2009; 11(1): 3-14.

37. Powell KP, Christianson CA, Cogswell WA, et al. Educational needs of primary care physicians regarding direct-to-consumer genetic testing. J Genet Couns 2012; 21(3): 469-478.

38. Humphries SE, Drenos F, Ken-Dror G, Talmud PJ. Coronary heart disease risk prediction in the era of genome-wide association studies: current status and what the future holds. Circulation 2010: 121(20): 2235-2248. 